\title{
Reactive Compensator Synthesis in Time-Domain as an Alternative to Harmonic Method
}

\author{
Maciej Siwczyński, Marcin Jaraczewski \\ Electrical and Computer Engineering, Cracow University of Technology, Kraków, Poland \\ Email: e-3@pk.edu.pl
}

Received July 6, 2011; revised March 1, 2012; accepted March 8, 2012

\begin{abstract}
The source reactive-current compensation is crucial in energy transmission efficiency. The compensator design in frequency-domain was already widely discussed and examined. This paper presents results of a study on how to design reactive compensators in time-domain. It's the first time the reactive compensator has been designed in time domain. The example of compensator design was presented.
\end{abstract}

Keywords: Power and Energy Theory; Reactive Power Filters; Reactive Current; Optimization; Harmonic Filter Design

\section{Introduction}

This article is a discussion on issue raised in the article of L. S. Czarnecki [1] where the author consider if it is possible to make current decomposition into active, reactive and unbalanced current in time domain and basing on it build reactive compensators. The current decomposition in time domain was presented in the previous articles [2, $3]$ and in this article is presented the reactive compensators design in time-domain.

\section{Reactive Current Compensation in Time-Domain}

In the article [3] was shown that source-receiver current can be decomposed into active and reactive current in " $s$ " domain i.e. for Laplace transform of signals. Reactive current can be compensated with the reactive compensator. The source-receiver current decomposition is given below:

$$
I(s)=G^{o}(s) E(s)+B^{o}(s) E(s)=I_{G}^{o}(s)+I_{B}^{o}(s)
$$

where:

$$
\begin{aligned}
& G^{o}(s)=\frac{1}{2}\left(Y^{o}(s)+Y^{o}(-s)\right) \\
& B^{o}(s)=\frac{1}{2}\left(Y^{o}(s)-Y^{o}(-s)\right)
\end{aligned}
$$

stand for the active and reactive parts of receiver admittance operator $Y^{\circ}(s)$.

The $I(s)$ transform for T-periodic signals is derived using the following relation between non-periodic and periodic signals transform [3].

$$
\frac{1}{\sigma+s} \rightarrow \frac{e^{-\sigma t}}{1-e^{-\sigma T}} \quad \frac{1}{\sigma-s} \rightarrow \frac{e^{\sigma(t-T)}}{1-e^{-\sigma T}}
$$

where $t \in[0, T), \operatorname{Re}(\sigma)>0$, T-time period.

It can be also calculated directly in time-domain as the T-periodic convolution:

$$
i(t)=\left(g^{o}(t)+b^{o}(t)\right) \otimes e(t)
$$

where $g^{o}(t), b^{o}(t)$ stands for T-periodic impulse response of admittance active and reactive part.

Connecting in parallel the compensator (Figure 1) the reactive current balance in time-domain states that:

$$
b^{k}(t)+b^{o}(t)=0
$$

\section{T-Periodic Impulse Response of Compensator and Receiver}

Admittance of the elementary RLC compensator branch

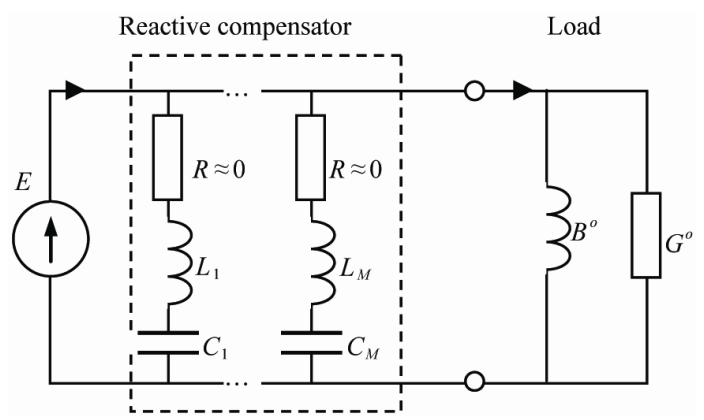

Figure 1. The zero impedance source with receiver and almost lossless compensator connected in parallel. 
is:

$$
Y^{k}(s)=\frac{1}{L} \frac{s}{\omega_{o}^{2}+2 \in s+s^{2}} ; \omega_{o}=\frac{1}{\sqrt{L C}} ; \in=\frac{R}{2 L}
$$

(We assume later that the compensator is composed of almost lossless elementary branches). And its reactive part is then (3)

$$
\begin{aligned}
B^{k}(s) & =\frac{1}{2 L}\left(\frac{s}{\omega_{o}^{2}+2 \in s+s^{2}}+\frac{s}{\omega_{o}^{2}-2 \in s+s^{2}}\right) \\
& =\frac{1}{2 L}\left(\frac{s}{(a+s)\left(a^{*}+s\right)}+\frac{s}{(a-s)\left(a^{*}-s\right)}\right)
\end{aligned}
$$

which leads to general form

$$
B(s)=\frac{d(-a)}{a+s}+\frac{d\left(-a^{*}\right)}{a^{*}+s}+\frac{d(a)}{a-s}+\frac{d\left(a^{*}\right)}{a^{*}-s}=\frac{L(s)}{M(s)}
$$

where $L(s), M(s)$ —odd and even polynomials.

The residues meet the relations: if

$$
d(-a)=\left.[B(s)(a+s)]\right|_{s=-a}=\frac{L(-a)}{M^{\prime}(-a)}=\frac{L(a)}{M^{\prime}(a)} \equiv d
$$

then:

$$
d(a)=-d ; d\left(-a^{*}\right)=d^{*} ; d\left(a^{*}\right)=-d^{*}
$$

where:

$M^{\prime}(s)$ is the derivative of $M(s)$ with respect to $s, d-$ real number.

Thus (7) reduces to

$$
B(s)=d\left(\frac{1}{a+s}-\frac{1}{a-s}\right)+d^{*}\left(\frac{1}{a^{*}+s}-\frac{1}{a^{*}-s}\right)
$$

and under (4) and trigonometric identity

$$
\operatorname{sh}(\alpha+j \beta)=\operatorname{sh}(\alpha) \cos (\beta)+j \operatorname{ch}(\alpha) \sin (\beta)
$$

where $a T=\alpha+j \beta$.

We get

$$
\begin{aligned}
b^{k}(t)=-2 d \operatorname{Re} & \left\{\frac{\operatorname{sh}\left(\alpha\left(\frac{t}{T}-\frac{1}{2}\right)\right) \cos \left(\beta\left(\frac{t}{T}-\frac{1}{2}\right)\right)}{\operatorname{sh}\left(\frac{\alpha}{2}\right) \cos \left(\frac{\beta}{2}\right)+j \operatorname{ch}\left(\frac{\alpha}{2}\right) \sin \left(\frac{\beta}{2}\right)}\right. \\
& \left.+j \frac{\operatorname{ch}\left(\alpha\left(\frac{t}{T}-\frac{1}{2}\right)\right) \sin \left(\beta\left(\frac{t}{T}-\frac{1}{2}\right)\right)}{\operatorname{sh}\left(\frac{\alpha}{2}\right) \cos \left(\frac{\beta}{2}\right)+j \operatorname{ch}\left(\frac{\alpha}{2}\right) \sin \left(\frac{\beta}{2}\right)}\right\}
\end{aligned}
$$
0 .

In the case of almost lossless compensator i.e. for $\alpha \rightarrow$

$$
b^{k}(t)=-2 d \frac{\sin \left(\beta\left(\frac{t}{T}-\frac{1}{2}\right)\right)}{\sin \left(\frac{\beta}{2}\right)} \quad t \in(0, T)
$$

where:

$$
\beta=\omega_{o} T=2 \pi \sqrt{\frac{X_{C}}{X_{L}}}
$$

$X_{C}=\frac{T}{2 \pi C}, \quad X_{L}=\frac{2 \pi L}{T}$ - capacitive and inductive reactance for the main frequency $f=1 / T$.

Residue for $B^{k}(s)$ in $\alpha=\epsilon+j \omega_{o} \approx j \omega_{o}$ can be calculated as

$$
d=\left.\left[B^{k}(s)(a+s)\right]\right|_{\left(\begin{array}{c}
s=-a \\
\epsilon=0
\end{array}\right)}=\frac{1}{2 L}
$$

Thus the T-periodic impulse response of reactive part of the elementary RLC branch (without $R$ ) has form

$$
b^{k}(t)=-\frac{1}{L} \frac{\sin \left(2 \pi \sqrt{\frac{X_{C}}{X_{L}}}\left(\frac{t}{T}-\frac{1}{2}\right)\right)}{\sin \left(\pi \sqrt{\frac{X_{C}}{X_{L}}}\right)} \quad \frac{t}{T} \in[0,1]
$$

where:

$$
\omega_{o m}, W_{m}=\sqrt{\left(\frac{X_{C}}{X_{L}}\right)_{m}}=\frac{\omega_{o n}}{(2 \pi) /(T)} \text {-angular and rela- }
$$

tive resonance frequency of $m$-th branch, and is depicted in Figure 2.

Later, in the article, it assumes that the reactive part of receiver $Y^{o}(s)$ has only real poles, so the receiver is nonoscillatory circuit not as the compensator.

For the single pole receiver e.g. $R_{L}$ or $R_{C}$ type the operational admittance is

$$
B^{o}(s)=b\left(\frac{1}{a+s}-\frac{1}{a-s}\right) \quad a>0
$$

thus its impulse response is

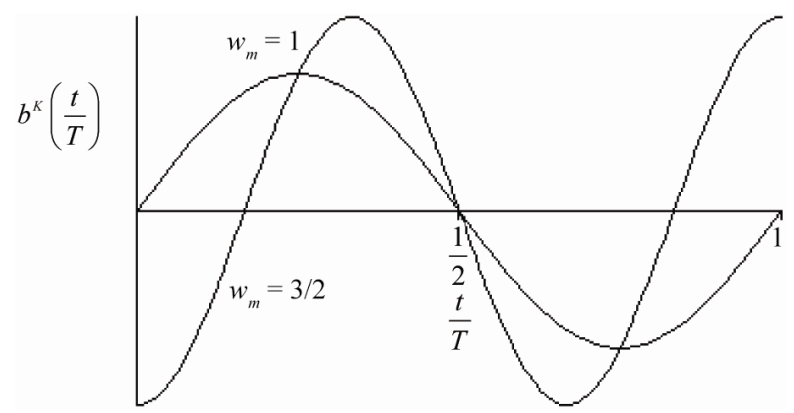

Figure 2. T-periodic impulse response of the compensator elementary branch. 


$$
b^{o}\left(\frac{t}{T}\right)=b \frac{e^{-a t}-e^{a(t-T)}}{1-e^{-a T}}=-b \frac{\operatorname{sh}\left(A\left(\frac{t}{T}-\frac{1}{2}\right)\right)}{\operatorname{sh}\left(\frac{A}{2}\right)}
$$

where $A=a T, t / T \in[0,1)$.

The coefficient $b$ can be both positive and negative what is shown below for the RRLC receiver (see Figure 3).

Its operator admittance is

$Y^{o}(s)=\frac{1}{R+s L}+\frac{1}{R+\frac{1}{s C}}=2 b_{L} \frac{1}{a_{L}+s}-2 b_{C} \frac{1}{a_{C}+s}+\frac{1}{R}$

where $a_{L}=\frac{R}{L}, b_{C}=\frac{1}{R C}, b_{L}=\frac{1}{2 L}, b_{C}=\frac{a_{C}}{2 R}=\frac{1}{2 R^{2} C}$

Then the reactive part of $Y^{o}(s)$ is

$$
B^{o}(s)=b_{L}\left(\frac{1}{a_{L}+s}-\frac{1}{a_{L}-s}\right)-b_{C}\left(\frac{1}{a_{C}+s}-\frac{1}{a_{C}-s}\right)
$$

and its T-periodic impulse response is

$$
b^{o}\left(\frac{t}{T}\right)=-b_{L} \frac{\operatorname{sh}\left(A_{L}\left(\frac{t}{T}-\frac{1}{2}\right)\right)}{\operatorname{sh}\left(\frac{A_{L}}{2}\right)}+b_{C} \frac{\operatorname{sh}\left(A_{C}\left(\frac{t}{T}-\frac{1}{2}\right)\right)}{\operatorname{sh}\left(\frac{A_{C}}{2}\right)}
$$

where $A_{L}=a_{L} T, A_{C}=a_{C} T$.

For the receiver shown in Figure 4, the operational admittance is

$$
Y^{o}(s)=\frac{1}{\frac{1}{s C}+R+s L}=\frac{1}{L} \frac{s}{\omega_{0}^{2}+2 \alpha s+s^{2}}
$$

where $\omega_{0}=\frac{1}{\sqrt{L C}}, \alpha=\frac{R}{2 L}$ and for the positive poles

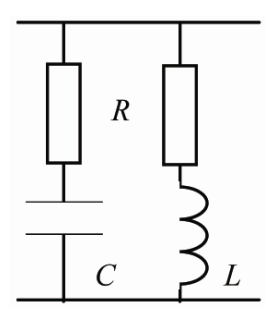

Figure 3. Example of the RRLC load.

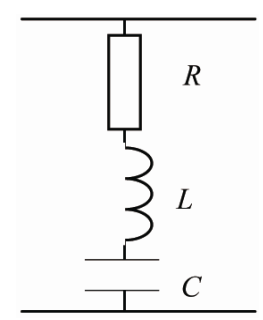

Figure 4. Example of the RRLC load. $\alpha^{2}-\omega^{2}>0, Y^{\circ}(s)$ takes form

$$
Y^{o}(s)=\frac{1}{L} \frac{s}{\left(a_{1}+s\right)\left(a_{2}+s\right)}=2\left(\frac{b_{1}}{a_{1}+s}+\frac{b_{2}}{a_{2}+s}\right)
$$

where $\alpha_{1}=\alpha+\sqrt{\alpha^{2}-\omega_{0}^{2}}, \quad \alpha_{2}=\alpha-\sqrt{\alpha^{2}-\omega_{0}^{2}}$ and the coefficients $b_{1}, b_{2}$ are then

$$
\begin{gathered}
b_{1}=\left.\frac{1}{2}\left[Y(s)\left(\alpha_{1}+s\right)\right]\right|_{s=-a_{1}}=\frac{1}{2 L} \frac{a_{1}}{a_{1}-a_{2}} \\
b_{2}=\left.\frac{1}{2}\left[Y(s)\left(\alpha_{2}+s\right)\right]\right|_{s=-a_{2}}=-\frac{1}{2 L} \frac{a_{2}}{a_{1}-a_{2}}
\end{gathered}
$$

thus

$$
B^{o}(s)=b_{1}\left(\frac{1}{a_{1}+s}-\frac{1}{a_{1}-s}\right)+b_{2}\left(\frac{1}{a_{2}+s}-\frac{1}{a_{2}-s}\right)
$$

The reactive function $b^{o}\left(\frac{t}{T}\right)$ (12) is shown in Fig-

ure 5. It is necessary to distinguish the two cases when $b$ $>0$ and $b<0$. When $A / 2 \rightarrow 0$ the curve become straight line.

$$
b^{o}\left(\frac{t}{T}\right)=-2 b\left(\frac{t}{T}-\frac{1}{2}\right) \quad \frac{t}{T} \in[0,1]
$$

because $\operatorname{sh}(x) \rightarrow 0$ for $x \rightarrow 0$.

Thus we arrive to the compensatory balance Equation (6) in a new form

$$
\sum_{m=1}^{M} \frac{1}{L_{m}} \frac{\sin \left(2 \pi w_{m}\left(\frac{t}{T}-\frac{1}{2}\right)\right)}{\operatorname{in}\left(\pi w_{m}\right)}=b^{o}\left(\frac{t}{T}\right)
$$

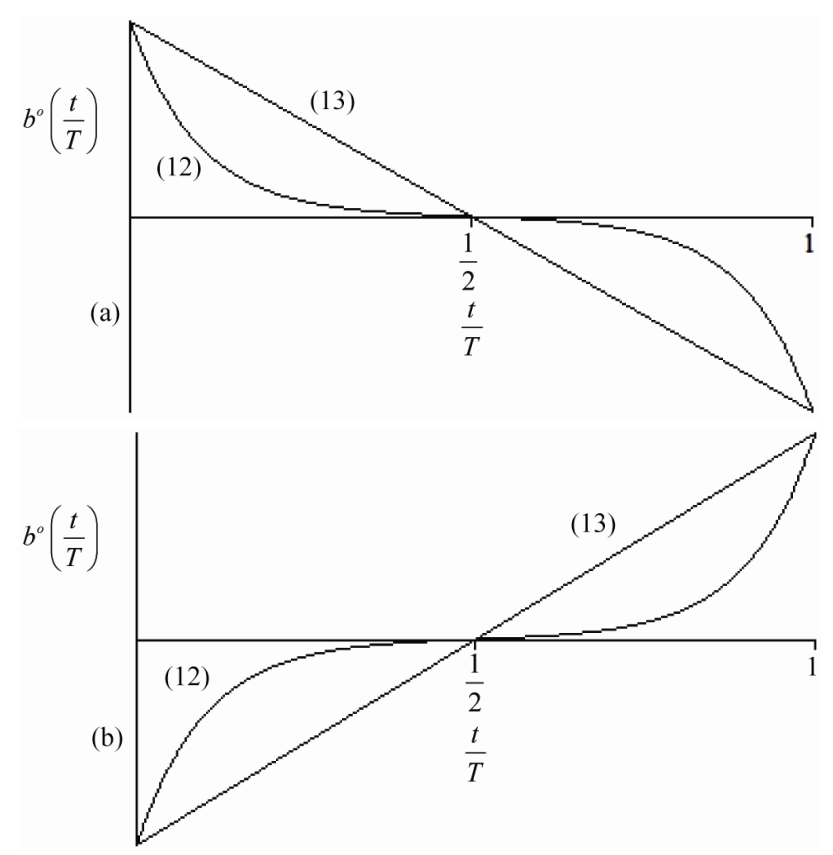

Figure 5. T-periodic single pole reactive operator impulse response of the load (a) $R L(b>0)$; (b) $R C(b<0)$. 
where $M$-total number of compensator branches.

The solution of (14) (see Figures 6 and 7) for the unknowns $L_{m}$ and $C_{m}$, can by find with optimization method. The (14) can be rewritten in respect to $D_{m}$ and $w_{m}$

$$
\sum_{m=1}^{M} D_{m} \sin \left(2 \pi w_{m}\left(\frac{t}{T}-\frac{1}{2}\right)\right)=b^{o}\left(\frac{t}{T}\right)
$$

where

$$
D_{m}=\frac{1}{L_{m} \sin \left(\pi w_{m}\right)}, \quad w_{m}=\sqrt{\left(\frac{X_{C}}{X_{L}}\right)_{m}}
$$

The relative frequencies of $L_{C}$ compensator branches have to meet the condition

$$
\sin \left(\pi w_{m}\right) \neq 0
$$

Thus we must choose relative resonance frequency $w_{m}$ of compensator branches as not the even numbers $w_{m} \neq p ; p$-even number.

The set of Equations (14) can be then solved for $D_{m}$ by minimizing $\delta^{2}$.

$$
\delta^{2} \rightarrow \min
$$

where

$$
\delta^{2}=\int_{0}^{1}\left(\sum_{m=1}^{M} D_{m} \min \left(2 \pi w_{m}\left(\frac{t}{T}-\frac{1}{2}\right)\right)-b^{o}\left(\frac{t}{T}\right)\right)^{2} \mathrm{~d}\left(\frac{t}{T}\right)
$$

After equate to zero appropriate partial derivatives

$$
\frac{\partial \delta}{\partial D_{m}}=0
$$

and assuming that

$$
w_{m}=m+\alpha, \quad m=1,2,3, \cdots ; 0<\alpha<1
$$

we get necessary minimum condition in form of the set of $M$ linear equations for $D_{m}$

$$
\begin{aligned}
& D_{m}-\sum_{n=1}^{M} D_{n} \frac{(-1)^{n+m} \sin (2 \pi \alpha)}{\pi(m+n+2 \alpha)} \\
= & 2 \int_{0}^{1} b^{o}\left(\frac{t}{T}\right) \sin \left(2 \pi w_{m}\left(\frac{t}{T}-\frac{1}{2}\right)\right) \mathrm{d}\left(\frac{t}{T}\right)
\end{aligned}
$$

Then we use relation $D_{m}=\frac{1}{L_{m} \sin \left(\pi w_{m}\right)}$ as to calculate $L_{m}$ of compensator branches.

The offset $\alpha$ in (17) must be less then 0.5 as to assure $L_{p}$ positive.

\section{Frequency-Domain Compensator Design}

The frequency-domain approach is a well known method (see M. Pasko [4-6]).

The counterpart of (6) in frequency-domain is

$$
B_{n}^{k}+B_{n}^{o}=0
$$

where:

$B_{n}^{k}, B_{n}^{o}$ - frequency response of compensator and receiver susceptances,

$n=1,2,3 \cdots$ - harmonic number.

For the elementary compensator branch ( $L_{C}$ in series) the branch elementary susceptance is

$$
B_{n}=\frac{n}{n^{2}-\frac{X_{C}}{X_{L}}} \frac{1}{X_{L}}
$$

Thus the formula of reactances balance (19) (see Figures 6 and 7) takes form

$$
\sum_{m=1}^{M} \frac{n}{n^{2}-w_{m}^{2}} \frac{1}{L_{m}}=\frac{2 \pi}{T} B_{n}^{o}
$$

The Equation (20) is the counterpart of (11) transformed to optimization task (18).

Then in the particular case of the $R_{L}$ in series receiver we get the set of $M$ linear equations for all $L_{m}$ of compensator branches

$$
\frac{T}{2 \pi} \sum_{m=1}^{M} \frac{1}{L_{m}} \frac{n}{\left(n^{2}-w_{m}^{2}\right)}=-\frac{X_{L} n}{R^{2}+X_{L}^{2} n^{2}}=B_{n}^{o}
$$

where

$n$-harmonic number,

$m$-branch number.

Comparing (18) with (21) we can see that both formulas are the system of linear equations, but in (18) we have
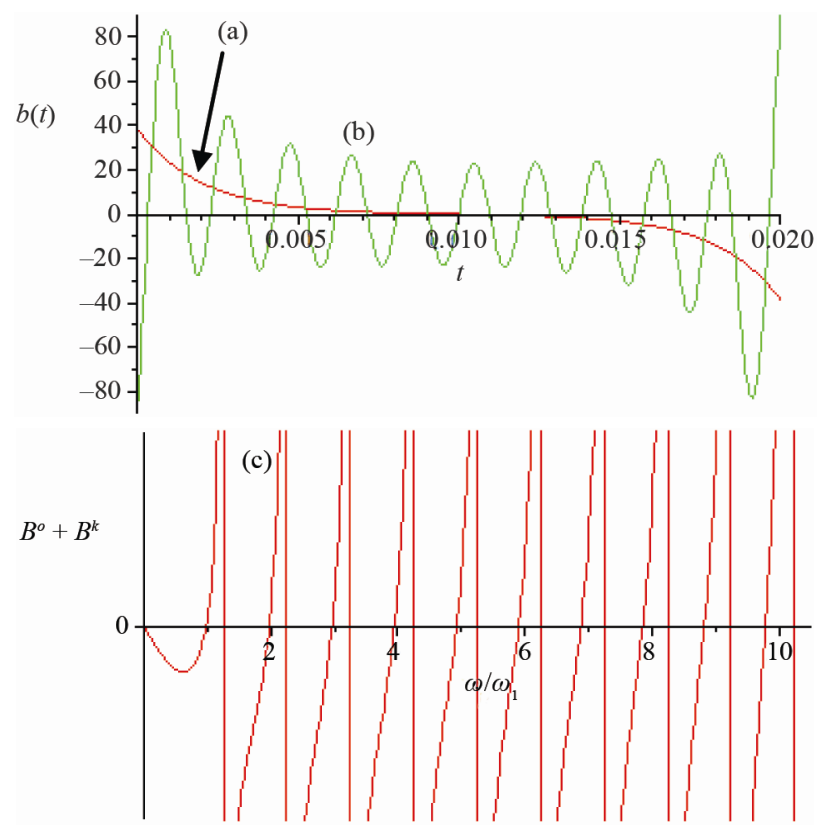

Figure 6. T-periodic time-domain response: (a) Receiver $b^{o}(t)$; (b) Compensator $\left(-b^{k}(t)\right)$ and frequency response; (c) $B^{o}\left(\omega / \omega_{1}\right)+B^{k}\left(\omega / \omega_{1}\right)$, for $\alpha=1 / 4$. 

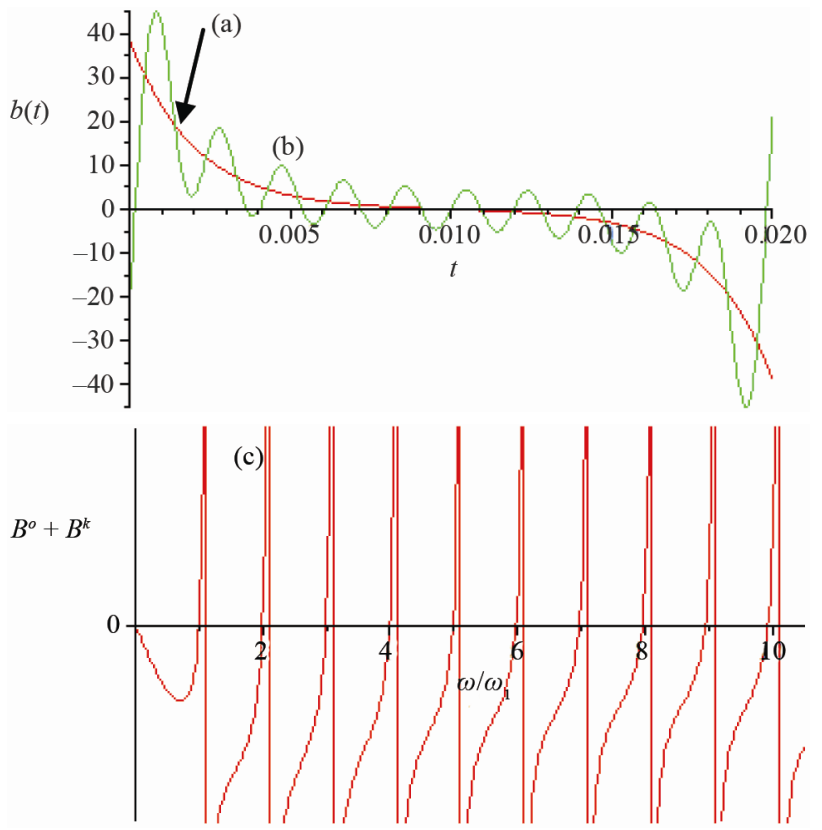

Figure 7. T-periodic time-domain response: (a) Receiver $b^{o}(t)$; (b) Compensator $\left(-b^{k}(t)\right)$ and frequency response; (c) $B^{o}\left(\omega / \omega_{1}\right)+B^{k}\left(\omega / \omega_{1}\right)$, for $\alpha=1 / 10$.

the impulse response instead of the frequency response of the receiver.

\section{Calculation Example}

Let consider the $R L$ load in series for which: $P=500$ [W], $T=0.02[\mathrm{~s}], \omega_{1}=314[\mathrm{rad} / \mathrm{s}], A L=T / \tau=10, L_{o}=25.7$ $[\mathrm{mH}]$ and $M=10, \tau$-time-constant of the load.

Effective compensation is up to 5 -th harmonic (see Figures 6 and 7).

The integral in the right side of (18) was calculated numerically using 21 samples and time samples was shifted by $T / 21 / 2$ due to singularity problem.

\section{Conclusion}

The frequency response method used until now to syn- thesis $L_{C}$ compensators and considered the only one [1], has its counterpart in time-domain. In both approaches the $L_{C}$ parameters can be found with simple optimization techniques for linear system. The only difference is that in (18) we can use directly the impulse response of the load (differentiated step response) instead of harmonic analysis. Moreover it is the first time in literature that the time-domain reactive compensator design is presented.

\section{REFERENCES}

[1] L. S. Czarnecki, "Discussion on 'a Uniform Concept of Reactive Power of Nonsinusoidal Currents in a TimeDomain'," Electrical Review, Vol. 85 No. 6, 2009, CDROM.

[2] M. Siwczyński and M. Jaraczewski, "The L1-Impulse Method as an Alternative to the Fourier Series in the Power Theory of Continues Time Systems," Bulletin of the Polish Academy of Science, Technical Sciences, Vol. 57, No 1, 2009, pp. 79-86.

[3] M. Siwczyński, "Decompositions: Active Current, Scattered Current, Reactive Current in Time-Domain-Single-Phase Circuits," Electrical Review, Vol. 6, 2010, pp. 11-17.

[4] M. Pasko, "Choice of the Two-Terminal Components, Compensating Reactive Current Component of the Linear Load Fed with Distorted Voltage," Seminarium z Podstaw Elektrotechniki $i$ Teorii Obwodow, Gliwice-Wisła, 20-23 May 1992, CD-ROM.

[5] M. Pasko, "Improvement of the Working Conditions of the Real Distorted Voltage Sources Using Two-Terminal LC components," Scientific Notebook of the Silesium University of Technology Electrotechniques, Vol. 117, 1991, pp. 45-62.

[6] M. Pasko and J. Walczak, "Optimization of the Power Quality Coefficients of the Electrical Circuits with Nonsinusoidal Periodic Signals," Wydawnictwo Politechniki Śląskiej, Gliwice, 1996. 